\title{
Whole genome sequencing Mycobacterium tuberculosis directly from sputum identifies more genetic diversity than sequencing from culture
}

\author{
Camus Nimmo ${ }^{1,2^{*}}$ (D) Liam P. Shaw ${ }^{3,4}$, Ronan Doyle ${ }^{1,5}$, Rachel Williams ${ }^{1}$, Kayleen Brien², Carrie Burgess', \\ Judith Breuer ${ }^{1}$, Francois Balloux ${ }^{3}$ and Alexander S. Pym ${ }^{2}$
}

\begin{abstract}
Background: Repeated culture reduces within-sample Mycobacterium tuberculosis genetic diversity due to selection of clones suited to growth in culture and/or random loss of lineages, but it is not known to what extent omitting the culture step altogether alters genetic diversity. We compared $M$. tuberculosis whole genome sequences generated from 33 paired clinical samples using two methods. In one method DNA was extracted directly from sputum then enriched with custom-designed SureSelect (Agilent) oligonucleotide baits and in the other it was extracted from mycobacterial growth indicator tube (MGIT) culture.
\end{abstract}

Results: DNA directly sequenced from sputum showed significantly more within-sample diversity than that from MGIT culture (median 5.0 vs 4.5 heterozygous alleles per sample, $p=0.04$ ). Resistance associated variants present as HAs occurred in four patients, and in two cases may provide a genotypic explanation for phenotypic resistance.

Conclusions: Culture-free M. tuberculosis whole genome sequencing detects more within-sample diversity than a leading culture-based method and may allow detection of mycobacteria that are not actively replicating.

Keywords: Mycobacterium tuberculosis, Drug-resistant tuberculosis, Whole genome sequencing, Sputum, Within-patient diversity, Heteroresistance

\section{Background}

International efforts to reduce tuberculosis (TB) infections and mortality over the last two decades have only been partially successful. In 2017, 10 million people developed TB and it has overtaken HIV as the infectious disease responsible for the most deaths worldwide [1,2]. Drug resistance is a major concern with a steady rise in the number of reported cases globally and rapid increases in some areas [1]. Patients with Mycobacterium tuberculosis resistant to the first line drugs rifampicin and isoniazid are classed as having multidrug-resistant (MDR) TB and usually treated with a standardised

\footnotetext{
* Correspondence: c.nimmo.04@cantab.net

${ }^{1}$ Division of Infection and Immunity, University College London, London WC1E 6BT, UK

${ }^{2}$ Africa Health Research Institute, Durban, South Africa

Full list of author information is available at the end of the article
}

second line drug regimen for at least 9 months, which is also used for rifampicin monoresistance [3, 4]. With the emergence of resistance to fluoroquinolones and aminoglycosides (extensively drug-resistant [XDR] TB) there is an increasing need for individualised therapy based on drug susceptibility testing (DST). Individualised therapy ensures patients are treated with sufficient active drugs which can prevent selection of additional resistance, improve treatment outcomes and reduce duration of infectiousness [5-8].

Traditionally, phenotypic culture-based DST was used to identify drug resistance but this is being replaced by rapid genetic tests that detect specific drug resistanceconferring mutations. Next generation whole genome sequencing (WGS) of M. tuberculosis is being increasingly used in research and clinical settings to comprehensively 
identify all drug resistance associated mutations [9]. $M$. tuberculosis has a conserved genome with little genetic diversity between strains and no evidence of horizontal gene transfer [10], but more detailed analysis of individual patient samples with WGS has identified genetically separate bacterial subpopulations in sequential sputum samples [11-16] and across different anatomical sites [17]. This within-patient diversity can occur as a result of mixed infection with genetically distinct strains or within-host evolution of a single infecting strain [18].

Bacterial subpopulations can be detected in clinical samples after sequencing reads are mapped to a reference genome where multiple base calls are detected at a single genomic site. These heterozygous alleles (HAs) at sites associated with drug resistance (resistance associated variants, RAVs) may reflect heteroresistance, where a fraction of the total bacterial population is drug susceptible while the remainder is resistant [19]. Identification of genetic diversity within clinical samples may improve detection of RAVs over currently available rapid genetic tests [19] and can be achieved with freely available WGS analysis toolkits [20-22]. Identifying RAVs could improve individualised therapy, prevent acquired resistance [12], and give insight into bacterial adaptation to the host.

M. tuberculosis WGS is usually performed on fresh or stored frozen cultured isolates to obtain sufficient purified mycobacterial DNA [23, 24]. However, the culture process can change the population structure from that of the original sample due to genetic drift (random loss of lineages) and/or the selection of subpopulations more suited to growth in culture [25-27]. Repeated subculture leads to loss of genetic diversity and heteroresistance [28]. Additionally, in the normal course of M. tuberculosis infection, some bacteria exist as viable non-culturable persister organisms that are hypothesised to cause the high relapse rate seen following treatment of insufficient duration. Although these organisms may be identified in sputum by techniques such as reporter phages or culture with resuscitation promoting factors [29, 30] they are likely to be missed by any sequencing method reliant on standard culture.

WGS directly from sputum without enrichment is challenging [23]. It has recently been improved by depleting human DNA during DNA extraction [31]. We have previously reported the use of oligonucleotide enrichment technology SureSelect (Agilent, CA, USA) to sequence $M$. tuberculosis DNA directly from sputum [32] and demonstrated its utility in determining a rapid genetic drug resistance profile [33, 34].

It remains unclear to what extent WGS of cultured $M$. tuberculosis samples underestimates the genetic diversity of the population in sputum samples. One previous study of 16 patients did not identify increased genetic diversity in $M$. tuberculosis DNA sequenced directly from sputum compared to DNA from culture [31], whereas another study of mostly drug susceptible patients showed sequencing directly from sputum identified a slight excess of HAs relative to culture [33]. Here we reanalyse heterozygous alleles (HAs) for the 12 available paired sequences with $>60$-fold mean genome coverage from that study [33] in addition to 21 newly collected samples from patients with MDR-TB and further explore the genomic location of the additional diversity identified.

\section{Results}

\section{Patient characteristics and drug susceptibility testing}

Whole genome sequences were obtained for 33 patients from both mycobacterial growth indicator tube (MGIT) culture and direct sputum sequencing. The patients were predominantly of black African ethnicity (83\%) and 50\% were HIV positive. First line phenotypic drug susceptibility testing (DST) results identified 20 patients with MDR-TB and one with rifampicin monoresistance. In addition there were two isoniazid monoresistant patients and ethambutol resistance was detected in 7 patients. Second-line phenotypic DST was performed for patients with rifampicin-resistant or MDR-TB and identified one case of kanamycin resistance (Table 1).

All samples had mean genome coverage of $60 x$ or above with at least $85 \%$ of the genome covered at $20 x$ (Additional file 1: Table S1). We observed greater mean coverage depth in sputum-derived sequences than MGIT sequences (median 173.7 vs 142.4, $p=0.03$, Additional file 1: Table S1), and so mapped reads were randomly downsampled to give equal mean coverage depth in each pair. A genotypic susceptibility profile was determined by evaluating MGIT WGS for consensus-level RAVs using a modified version of publicly available lists [22, 35]. Genotypic RAVs predicted all rifampicin phenotypic resistance and $>95 \%$ of isoniazid phenotypic resistance. Ethambutol genotypic RAVs were poorly predictive of phenotypic resistance in line with findings from other studies [36] (Table 1). The patient with kanamycin phenotypic resistance was correctly identified by an $r$ rs a1401g RAV. No full phenotypic fluoroquinolone phenotypic resistance was identified, but several colonies from patient F1013 did grow in the presence of ofloxacin (although not enough to be classified as resistant). The consensus sequences from this patient harboured a $\operatorname{gyr} B$ E501D mutation which is believed to confer resistance to moxifloxacin but not other fluoroquinolones, which may explain the borderline phenotypic DST result [37].

\section{Genetic diversity}

To compare consensus sequences from sputum and MGIT, a WGS consensus sequence-level maximum likelihood 
Table 1 Phenotypic and genotypic drug susceptibility testing (DST) results and sensitivity and specificity of genotypic DST relative to phenotypic DST

\begin{tabular}{lllll}
\hline Drug & Resistance by phenotypic DST & Resistance by genotypic DST & Genotypic DST sensitivity & Genotypic DST specificity \\
\hline $\begin{array}{llll}\text { First line drugs } \\
\text { Rifampicin }\end{array}$ & $21 / 32(65.6 \%)$ & $21 / 33(63.6 \%)$ & $21 / 21(100 \%)^{\mathrm{a}}$ & $21 / 21(100 \%)$ \\
Isoniazid & $22 / 32(68.8 \%)$ & $24 / 36(66.7 \%)$ & $21 / 22(95.5 \%)$ & $23 / 24(95.8 \%)$ \\
Ethambutol & $7 / 31(22.6 \%)$ & $15 / 34(44.1 \%)$ & $7 / 7(100 \%)$ & $7 / 15(46.7 \%)$ \\
Second line drugs) & & & & $0 / 1(0 \%)^{b}$ \\
Ofloxacin & 0/22 (0.0\%) & $1 / 22(4.5 \%)$ & $\mathrm{N} / \mathrm{A}$ & $1 / 1(100 \%)$ \\
Kanamycin & $1 / 22(4.5 \%)$ & $1 / 22(4.5 \%)$ & $1 / 1(100 \%)$ &
\end{tabular}

Phenotypic DST available for first line drugs for 32 of the 33 patients, and for second line drugs for 22 patients who demonstrated rifampicin drug resistance ${ }^{a}$ In one directly-sequenced sputum samples rifampicin RAVs were missed due to low coverage, although they were identified in the corresponding MGIT sample ${ }^{\mathrm{b}}$ This sample had $<1 \%$ of colonies grow in the presence of ofloxacin, so is categorised as susceptible but may have low-level or heteroresistance to fluoroquinolones (see main text)

phylogenetic tree was constructed (Additional file 1: Figure S1). As expected, all paired sequences were closely related, with a median difference of 0.0 (range 0-1) single nucleotide polymorphisms (SNPs). Samples from patients F1066 and F1067 were closely related with only one consensus-level SNP separating all four consensus sequences. There was no obvious epidemiological link between these patients (although this study was not designed to collect comprehensive epidemiological information) and they lived $20 \mathrm{~km}$ apart in Durban. However, both patients were admitted contemporaneously to an MDR treatment facility and sampled on the same day. DNA extraction and sequencing occurred on different runs. Therefore the close genetic linkage may represent direct transmission within a hospital setting, a community transmission chain or an unlikely cross-contamination during sample collection.

Having established congruence between sputum and MGIT sequences at the consensus level we then compared genetic diversity by DNA source. We first defined a threshold for calling variants present as heterozygous alleles (HAs) in our entire dataset by using a range of minimum read count frequencies as described in the methods (Fig. 1). Below a minimum of three supporting reads there was an exponential increase in the number of HAs identified, which may be indicative of the inclusion of sequencing errors. To reduce this risk, we used a threshold of a minimum of four supporting reads.

Genetic diversity may occur because of within-host evolution or mixed infection. To identify mixed infection we used a SNP-based barcode [38] to scan all HAs for a panel of 413 robust phylogenetically informative SNPs that can resolve $M$. tuberculosis into one of seven lineages and 55 sub-lineages. We found three phylogenetic SNPs among the HAs. In all cases the heterozygous phylogenetic SNP originated from the same sublineage as other SNPs present at 100\% frequency, and there were no cases of HAs indicating the presence of more than one lineage or sublineage. We tested for mixed infection with the same sublineage by screening samples by HA frequency and then using Bayseian model based clustering in samples with $>10$ HAs as described previously [39]. This identified mixed infection in the sputum sample from patient F1096, which had 261 heterozygous alleles, greater than 10 times that in any other sample. This patient was therefore excluded from further analyses.

As a first step to comparing diversity between sputum and MGIT sequenced samples we looked at the location of genetic diversity within the $M$. tuberculosis genome. HAs were widely dispersed across the genome at similar sites in both sputum and MGIT samples. The genes with the greatest density of HAs are shown in Table 2 .

Notably, genetic diversity was found in the ribosomal RNA (rRNA) genes ( $r r s$ and $r r l$ ) uniquely in sputum samples, compared to other genes where distribution of diversity between MGIT and sputum was more balanced. As rRNA contains regions that are highly conserved across bacteria [40], we considered the possibility that

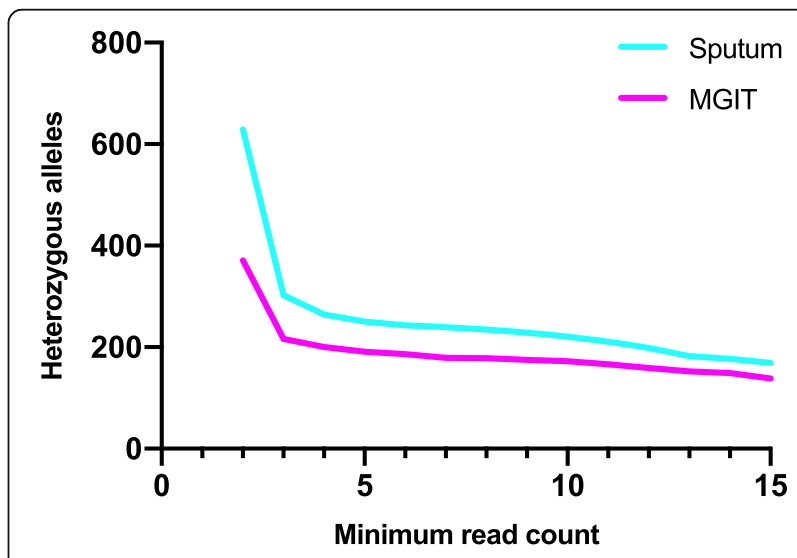

Fig. 1 Variation in total number of heterozygous alleles (HAs) identified across all 33 patients in sequences generated from sputum and MGIT depending on minimum supporting read count threshold 
Table 2 Genes with $\geq 2$ heterozygous alleles (HAs) across all sputum samples, ordered by greatest number of HAs per base

\begin{tabular}{|c|c|c|c|c|c|}
\hline \multirow[t]{2}{*}{ Gene } & \multicolumn{2}{|c|}{ Heterozygous alleles per base } & \multicolumn{2}{|c|}{ Total number of heterozygous alleles } & \multirow[t]{2}{*}{ Functional category } \\
\hline & Sputum & MGIT & Sputum & MGIT & \\
\hline rv1319c & 0.021 & 0.021 & 33 & 33 & Metabolism and respiration \\
\hline rrs & 0.016 & 0.000 & 25 & 0 & $16 \mathrm{~S}$ ribosomal RNA \\
\hline$r r l$ & 0.006 & 0.000 & 19 & 0 & $23 \mathrm{~S}$ ribosomal RNA \\
\hline ppsA & 0.003 & 0.001 & 15 & 4 & Lipid metabolism \\
\hline rv2082 & 0.006 & 0.006 & 13 & 14 & Unknown function \\
\hline accE5 & 0.006 & 0.000 & 3 & 0 & Lipid metabolism \\
\hline IppB & 0.005 & 0.005 & 3 & 3 & Probable surface lipoprotein \\
\hline pks 12 & 0.000 & 0.001 & 3 & 10 & Lipid metabolism \\
\hline rv2319c & 0.003 & 0.005 & 3 & 4 & Stress protein \\
\hline IppA & 0.003 & 0.002 & 2 & 1 & Probable surface lipoprotein \\
\hline rpoc & 0.001 & 0.001 & 2 & 3 & RNA polymerase beta' subunit \\
\hline rv3888c & 0.002 & 0.001 & 2 & 1 & Probable membrane protein \\
\hline $\operatorname{vap} C 25$ & 0.005 & 0.000 & 2 & 0 & Possible toxin \\
\hline $\operatorname{vap} C 31$ & 0.005 & 0.002 & 2 & 1 & Possible toxin \\
\hline
\end{tabular}

SureSelect baits targeting rRNA genes were capturing both M. tuberculosis and other bacterial species. To evaluate this, metagenomic taxonomic assignment was performed on all reads by sampling reads that were not assigned to $M$. tuberculosis (i.e. presumed contaminants from other bacteria). We then performed a BLAST search against the most diverse genes listed in Table 2 which indicated that a sizeable proportion of non- $M$. tuberculosis reads from directly sequenced sputum had a BLAST hit of at least 30 bases to M. tuberculosis rrs and $r r l$ genes that encode rRNA (330 BLAST hits from sputum sequences vs 4 BLAST hits from MGIT sequences, median $8.5 \%$ vs $0.0 \%, p<0.01$, Additional file 1: Figure S2). There were no BLAST hits against any of the other genes with $\geq 2$ sputum HAs apart from rpoC, for which there were 3 BLAST hits from sputum sequences but none from MGIT sequences (median $0.0 \%$ for both sputum and MGIT sequences), indicating that this issue appears largely specific to rRNA. To determine if contaminating reads were contributing to HAs identified in intergenic regions, we repeated this analysis for all intergenic regions with $\geq 2$ sputum HAs (Additional file 1: Table S2). There were no BLAST hits to any of these regions, suggesting that this is not the case. The taxonomic assignment of these contaminating reads were typical of genera composing the oral flora, with a high representation of Actinomyces, Fusobacterium, Prevotella, and Streptococcus (Additional file 1: Figure S3).

This supported the hypothesis that the baits may enrich rRNA from other organisms so rRNA genes were excluded from further analysis. The difference in diversity between sputum and MGIT sequences can be explained by the selective nature of MGIT media which will enrich $M$. tuberculosis sequences and the decontamination step used to kill non-mycobacteria prior to culture inoculation. Importantly the frequency of HAs in other highly diverse genes between sequencing strategies was more balanced (Table 2) in addition to the lack of BLAST hits of contaminating reads to these genes.

After excluding the sample with mixed infection and removing rRNA gene sequences we compared the frequency of HAs in sputum and MGIT. There were 265 HAs identified across all sputum samples compared to 200 in MGIT samples (median 5.0 vs $4.5, p=0.04$, Additional file 1: Table S1). In both sputum and MGIT samples, the majority of HAs were indels, and non-synonymous mutations were more commonly frameshift than missense mutations (Table 3). The distribution of HAs by patient is shown in Fig. 2.

\section{Genetic diversity in drug resistance genes}

HAs in drug resistance associated regions, including promoters and intergenic regions, were individually assessed. Four of the 32 patients with single strain infection had RAVs present as HAs in at least one gene, which are shown in Table 4. Patient F1002 had three compensatory mutations in rpoC present at HAs in both sequences. As described above, the strains from patients F1066 and F1067 were highly related with only one consensus SNP difference between all four sequences. Both had phenotypic high level isoniazid resistance with no consensus-level katG or inhA mutation, but had frameshift katG mutations present as HAs which have the potential to cause resistance [43]. F1066 and RF021 had $R v 1979 c$ and pncA mutations respectively at low 
Table 3 Variants identified in MGIT and sputum derived sequences from paired samples

\begin{tabular}{lll}
\hline & Sputum variants & MGIT variants \\
\hline Total variants & 24,480 & 25,465 \\
$\begin{array}{l}\text { Total variants present as HAs } \\
\text { (\% of total variants) }\end{array}$ & $265(1.1 \%)$ & $200(0.8 \%)$ \\
Median HAs per sample & 5.0 & 4.5 \\
Variant type (\% all HAs) & & \\
SNP & $217(81.9 \%)$ & $174(87.0 \%)$ \\
MNP & $2(0.8 \%)$ & $0(0.0 \%)$ \\
Insertion & $4(1.5 \%)$ & $1(0.5 \%)$ \\
Deletion & $24(9.1 \%)$ & $15(7.5 \%)$ \\
Complex & $18(6.8 \%)$ & $10(5.0 \%)$ \\
Coding change (\% all HAs) & & $77(38.5 \%)$ \\
Non-synonymous (missense) & $93(35.1 \%)$ & $7(3.5 \%)$ \\
Non-synonymous (frameshift) & $6(2.3 \%)$ & $57(28.5 \%)$ \\
Synonymous & $57(21.5 \%)$ & $59(29.5 \%)$ \\
Intergenic & $109(41.1 \%)$ &
\end{tabular}

Values given represent totals for 32 paired samples. SNP single nucleotide polymorphism, MNP multi-nucleotide polymorphism

frequency in sputum only which have the potential to confer phenotypic resistance to clofazimine (Rv1979c) and pyrazinamide $(p n c A)$, although no phenotypic testing was performed for these drugs.

\section{Discussion}

In this study we performed whole genome sequencing using DNA from sputum and MGIT culture in paired samples from 33 patients and compared within-patient genetic diversity between methods. All paired sequences were closely related at the consensus level, and WGS predicted phenotypic drug susceptibility with over 95\% sensitivity and specificity for rifampicin and isoniazid in line with published data [44].

We find that the rRNA genes have high levels of diversity in sputum samples, but believe this is due to non-mycobacterial DNA hybridising to the capture baits. This conclusion is borne out by the taxonomic assignment of reads aligning to these genes in common oral bacteria. We therefore excluded these from further analysis, and recommend others using enrichment from sputum do similarly. We find more diversity when sequencing directly from sputum with significantly more unique heterozygous alleles (HAs) than sequencing from MGIT culture $(p=0.04)$.

The understanding of within-patient M. tuberculosis genetic diversity is becoming increasingly important as the detection of rare variants has been shown to improve the correlation between phenotypic and genotypic drug resistance profiles [19] and can identify emerging drug resistance [11, 12]. Not including a culture step avoids the introduction of bias towards culture-adapted subpopulations and the impact of random chance and is also likely to incorporate DNA from viable nonculturable mycobacteria. A reduction in genetic diversity has previously been shown with sequential $M$. tuberculosis subculture [25, 28], but was not confirmed by a study performing WGS directly from sputum [31]. However, the 16 paired sputum and MGIT samples compared by Votintseva [31] had a minimum of $5 x$ coverage compared to a minimum $60 x$ coverage in this study, and were likely to contain less genetic

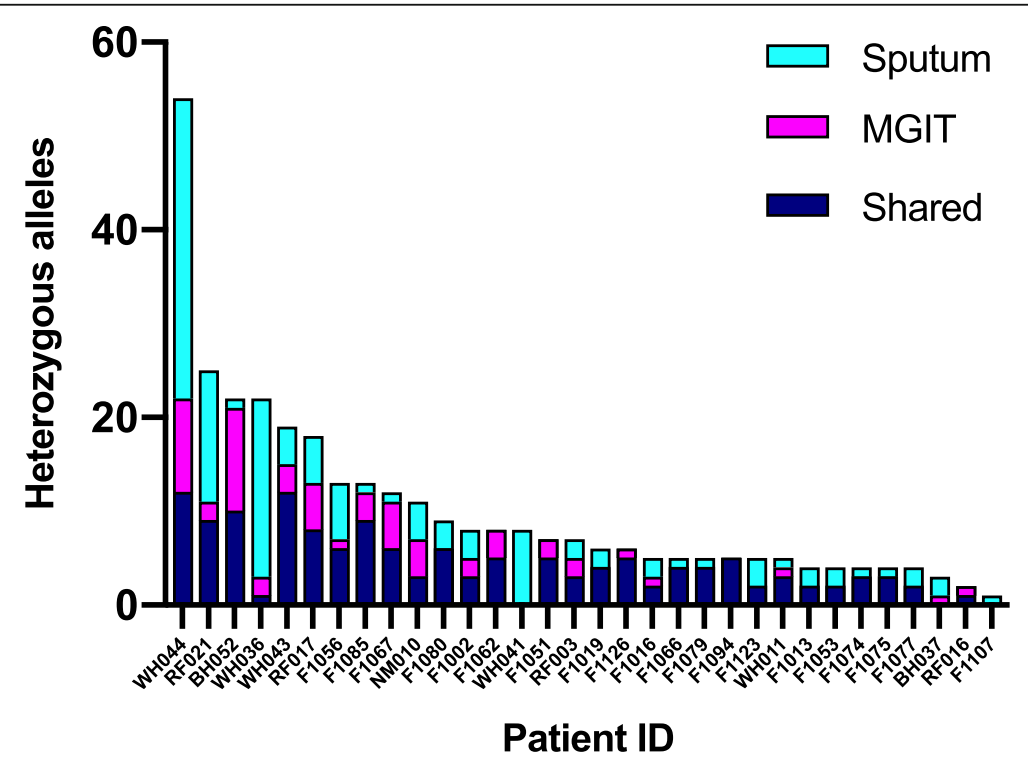

Fig. 2 Number of heterozygous alleles (HAs) found in directly sequenced sputum only (sputum), MGIT (MGIT) only or in both samples (shared) by patient 
Table 4 Resistance associated variants present as heterozygous alleles (HAs)

\begin{tabular}{lllll}
\hline Patient ID & Phenotypic resistance & Mutation & Frequency (MGIT/sputum) & Description \\
\hline F1002 & Rifampicin & rpoB S450 L & $100 \% / 100 \%$ & High confidence resistance mutation \\
F1002 & Rifampicin & $\operatorname{rpoC~G332R~[41]~}$ & $82.6 \% / 21.7 \%$ & Putative compensatory mutations \\
F1002 & Rifampicin & $\operatorname{rpoC~L516P~[41]~}$ & $12.7 \% / 7.7 \%$ & \\
F1002 & Rifampicin & $\operatorname{rpoC~P1040S~[42]~}$ & $21.7 \% / 12.3 \%$ & Possible resistance mutations, not \\
F1066 & Isoniazid (high) & $\operatorname{katG~N218fs}$ & $0.0 \% / 6.9 \%$ & previously described \\
F1066 & Clofazimine - not tested & Rv1979C G376D & $0.0 \% / 0.5 \%$ & $10.7 \% / 7.6 \%$ \\
F1067 & Isoniazid (high) & $\operatorname{katG~N218fs}$ & $0 \% / 2.5 \%$ & \\
RF021 & Pyrazinamide - testing failed & pncA Q122H & & \\
\hline
\end{tabular}

material as they were surplus clinical rather than dedicated research samples.

Two-thirds of the patients with MDR-TB had already been treated for drug susceptible-TB (DS-TB), and additional diversity in sputum samples may represent early adaptation to drug pressure. As direct sputum sequencing does not rely on live mycobacteria, DNA from recently killed $M$. tuberculosis is likely to also be sequenced, meaning that recent genomic mutations are likely to be represented as HAs.

In two patients, RAVs present as HAs provided a likely genotypic basis for otherwise unexplained phenotypic resistance. Given the small total number of resistance mutations in this study, it is not possible to draw conclusions about the frequency of heterozygous RAVs in directly sequenced sputum. However the presence of heterozygous RAVs in both MGIT and sputum sequences reinforces the biological importance of these mutations.

To reduce the risk of sample cross contamination, paired samples were extracted on different days, prepared in different sequencing libraries and sequenced on different runs. However it is not possible to completely exclude the possibility of contamination during sample collection and between different samples processed in batches. A further limitation of this study is that it can be difficult to distinguish low frequency variants from sequencing error. The SureSelect library preparation protocol for sputum sequencing incorporates more PCR cycles than that used for MGIT sequencing, which may increase the risk of error. Where possible this could be evaluated further by performing technical sequencing replicates on extracted DNA samples, although this was not possible due to insufficient surplus material and financial constraints. To reduce the risk of sequencing errors we used high read and mapping quality thresholds, and required a stringent $98 \%$ identity between sequenced reads and the reference genome. Low frequency variants of particular clinical importance could be confirmed by resequencing the same DNA samples.

\section{Conclusions}

Directly sequencing $M$. tuberculosis from sputum is able to identify more genetic diversity than sequencing from culture. Characterising within-patient genetic diversity is important to understand bacterial adaptation to drug treatment and the acquisition of drug resistance. It also has potential to identify low frequency RAVs that may further enhance the prediction of drug resistance phenotype from genotype.

\section{Methods}

\section{Patient enrolment}

Adult patients presenting with a new diagnosis of sputum culture positive TB were included in the study. Patients were recruited in London, UK $(n=12)$ and Durban, South Africa $(n=21)$. All patients recruited in Durban were Xpert MTB/RIF (Cepheid, CA, USA) positive for rifampicin resistance. Two sputum samples were collected prior to starting the current treatment regimen, with one inoculated into mycobacterial growth indicator tube (MGIT) culture (BD, NJ, USA) and the other used for direct DNA extraction. Therefore for patients with drug susceptible-TB (DS-TB), sputum was collected prior to taking any TB therapy, while patients starting MDR-TB treatment may have already taken treatment for DS-TB if this was intiated prior to resistance results being available.

\section{Ethics, consent and permissions}

All patients gave written informed consent to participate in the study. Ethical approval for the London study was granted by NHS National Research Ethics Service East Midlands-Nottingham 1 (reference 15/EM/0091). Ethical approval for the Durban study was granted by University of KwaZulu-Natal Biomedical Research Ethics Committee (reference BE022/13).

\section{Microbiology}

MGIT samples were incubated in a BACTEC MGIT 960 (BD, NJ, USA) until flagging positive. Phenotypic DST 
data for London samples were those provided to treating hospitals by Public Health England. Phenotypic DST were performed using equivalent standardised methods. For Durban samples this was the solid agar proportion method (Additional file 1: Methods) and for London samples the resistance ratio method [45].

\section{DNA extraction and sequencing}

Positive MGIT tubes were centrifuged at 16,000 $\mathrm{g}$ for 15 $\mathrm{min}$ and the supernatant removed. Cells were resuspended in phosphate-buffered saline before undergoing heat killing at $95^{\circ} \mathrm{C}$ for $1 \mathrm{~h}$ followed by centrifugation at $16,000 \mathrm{~g}$ for $15 \mathrm{~min}$. The supernatant was removed and the sample resuspended in $1 \mathrm{~mL}$ sterile saline $(0.9 \% \mathrm{w} /$ v). The wash step was repeated. DNA was extracted with mechanical ribolysis before purification with DiaSorin Liaison Ixt (DiaSorin, Italy) or CTAB [46]. NEBNext Ultra II DNA (New England Biolabs, MA, USA) was used for DNA library preparation.

Sputum samples for direct sequencing were heat killed, centrifuged at $16,000 \mathrm{~g}$ for $15 \mathrm{~min}$ and the supernatant was removed. DNA extraction was performed with mechanical ribolysis followed by purification using DiaSorin Liaison Ixt (DiaSorin, Italy) or DNeasy blood \& tissue kit (Qiagen, Germany) [46]. Target enrichment was performed using SureSelect with a custom-designed bait set covering the entire positive strand of the $M$. tuberculosis genome as described previously [33]. Batches of 48 multiplexed samples were sequenced on NextSeq 500 (Illumina, CA, USA) 300-cycle paired end runs with a mid-output kit. Sequencing was performed by the Pathogen Genomics Unit at University College London in a dedicated laboratory where one sequencing run was processed at a time. All paired samples were extracted, prepared and sequenced on different days. The National Center for Biotechnology Information Sequence Read Archive (NCBI SRA) accession number for each sample is shown in Additional file 1: Table S3.

\section{Read mapping}

DNA sequence reads were adapter and quality trimmed then aligned to the H37Rv reference genome (GenBank accession NC_000962.3) with Trim Galore v0.4.4 [47] and BBMap v38.32 [48], with mapped reads stored in an output bam file. Duplicate reads were removed with Picard tools v1.130 [49] MarkDuplicates and coverage statistics generated with Qualimap v2.2.1 [50]. For each sample pair, the bam file with greater mean genome coverage was randomly downsampled to that of the paired sample with Picard tools v1.130 [49] DownsampleSam. All further analyses were performed using these downsampled bam files. Command line parameters used are specified in the Additional file 1: Methods.

\section{Variant calling}

Variant calling for comparison for HA counts was performed with FreeBayes v1.2 [51]. Variants falling in or within 50 bases of PE/PPE family genes and repeat elements were excluded using vcfinteresect in vcflib [52]. For the initial analysis of genetic diversity, variants were included if supported by $\geq 2$ reads, with $\geq 1$ forward and reverse read, no read position bias, a minimum mapping quality of 30 and base quality of 30 . The minimum supporting read threshold was then increased in a stepwise fashion from 2 to 15 . Variant calling files where variants were supported $\geq 4$ supporting reads including $\geq 1$ forward and reverse read were used to compare HA frequency and location and to screen for mixed infection.

The phylogenetic tree was constructed by calling variants with VarScan v2.4.0 [53] mpileup2cns as this is able to generate consensus-level calls at each reference sequence base. SNPs were then used to generate a sequence of equal length to the reference using a custom perl script and these sequences were combined in a multi-alignment fasta file. SNP sites were extracted from this alignment using snp-sites v2.4.1 [54], and pairwise SNP differences calculated using snp-dists v0.6.3 [55]. Extracted SNP sites were used to generate a maximum likelihood phylogenetic tree using RaxML v8.2.12 [56] which was visualised using FigTree v1.4.3.

\section{Identification of mixed infection}

All samples were screened for evidence of mixed infection using described methods [39]. In brief, any sample with 10 or fewer heterozygous SNPs, or between 11 and 20 heterozygous SNPs where heterozygous SNPs were $\leq$ $1.5 \%$ of all SNPs was classified as not mixed. For other samples, the Baysian mixture model analysis [39] was used where samples with a Bayesian information criterion value $>20$ for presence of more than one strain were assumed to be mixed.

\section{Metagenomic assignment}

Sequencing reads were classified using Kraken v0.10.6 [57] against a custom Kraken database previously constructed from all available RefSeq genomes for bacteria, archaea, viruses, protozoa, and fungi, as well as all RefSeq plasmids (as of September 19th 2017) and three human genome reference sequences [58]. The size of the final database after shrinking was $193 \mathrm{~Gb}$, covering 38,190 distinct NCBI taxonomic IDs.

To assess the proportion of contaminating reads that could generate spurious diversity when mapped to $M$. tuberculosis ribosomal genes, we randomly subsampled 100 reads taxonomically assigned as non- $M$. tuberculosis and performed a BLAST search with 
blastn v2.2.28 [59] against each gene as described from the H37Rv reference genome. We only analysed hits of at least 30 bases.

\section{Statistics}

Statistical analyses were performed with Prism v8.0 (Graphpad, CA, USA). Mean coverage depth statistics, number of HAs and BLAST hits of contaminating reads in paired samples were compared using a two-tailed Wilcoxon matched-pairs signed rank test.

\section{Additional file}

Additional file 1: Supplemetary Methods, Figures and Tables. (PDF 490 $\mathrm{kb})$

\section{Abbreviations}

DST: Drug susceptibility testing; DS-TB: Drug susceptible-tuberculosis; HA: Heterozygous allele; MDR-TB: Multidrug resistant-tuberculosis; MGIT: Mycobacterial growth indicator tube; RAV: Resistance associated variant; rRNA: Ribosomal RNA; SNP: Single nucleotide polymorphism; TB: Tuberculosis; WGS: Whole genome sequencing

\section{Acknowledgements}

The authors would like to thank Sashen Moodley, Ashentha Govender and Colin Chetty in the Microbiology Core, Africa Health Research Institute.

\section{Funding}

Camus Nimmo is funded by a Wellcome Trust Research Training Fellowship reference 203583/Z/16/Z. Judith Breuer receives funding from the UCL/UCLH NIHR funded Biomedical Research Centre. This work was additionally funded by National Institute for Health Research via the UCLH/UCL Biomedical Research Centre (grant number BRC/176/II/JB/101350) and the PATHSEEK European Union's Seventh Programme for research and technological development (grant number 304875). The funding bodies had no input on study design, analysis, data interpretation or manuscript writing.

\section{Availability of data and materials}

Original fastq files are available at NCBI Sequence Read Archive with BioProject reference PRJNA486713: https://www.ncbi.nlm.nih.gov/bioproject/PRJNA486713/

\section{Authors' contributions}

Study conception: JB, ASP, Data collection: $\mathrm{CB}, \mathrm{KB}$, Analysis and interpretation: CN, LPS, RD, RW, Drafting of manuscript: CN, LPS, Revision of manuscript: FB, JB, ASP, Final approval of manuscript: CN, LPS, RD, RW, KB, CB, JB, FB, ASP.

\section{Ethics approval and consent to participate}

All patients gave written informed consent to participate in the study. Ethical approval for the London study was granted by NHS National Research Ethics Service East Midlands-Nottingham 1 (reference 15/EM/0091). Ethical approval for the Durban study was granted by University of KwaZulu-Natal Biomedical Research Ethics Committee (reference BE022/13).

\section{Consent for publication}

Not applicable

\section{Competing interests}

The authors declare that they have no competing interests.

\section{Publisher's Note}

Springer Nature remains neutral with regard to jurisdictional claims in published maps and institutional affiliations.

\section{Author details}

'Division of Infection and Immunity, University College London, London WC1E 6BT, UK. ${ }^{2}$ Africa Health Research Institute, Durban, South Africa. ${ }^{3} \mathrm{UCL}$
Genetics Institute, University College London, London WC1E 6BT, UK. ${ }^{4}$ Nuffield Department of Clinical Medicine, Oxford University, Oxford OX3 7BN, UK. ${ }^{5}$ Clinical Research Department, London School of Hygiene and Tropical Medicine, London WC1E 7HT, UK.

Received: 3 October 2018 Accepted: 7 May 2019

Published online: 20 May 2019

\section{References}

1. Global Tuberculosis Report 2018. Geneva: World Health Organization; 2018 2018

2. Murray CJ, Ortblad KF, Guinovart C, Lim SS, Wolock TM, Roberts DA, et al. Global, regional, and national incidence and mortality for HIV, tuberculosis, and malaria during 1990-2013: a systematic analysis for the global burden of disease study 2013. Lancet. 2014:384(9947):1005-70.

3. Dheda K, Gumbo T, Maartens G, Dooley KE, McNerney R, Murray M, et al. The epidemiology, pathogenesis, transmission, diagnosis, and management of multidrug-resistant, extensively drug-resistant, and incurable tuberculosis. Lancet Respir Med. 2017;5(4):291-360.

4. WHO treatment guidelines for drug-resistant tuberculosis. World Health Organization; 2016.

5. Trauner A, Liu Q, Via LE, Liu X, Ruan X, Liang L, et al. The within-host population dynamics of mycobacterium tuberculosis vary with treatment efficacy. Genome Biol. 2017:18(1):71.

6. Olaru ID, Lange C, Heyckendorf J. Personalized medicine for patients with MDR-TB. J Antimicrob Chemother. 2016;71(4):852-5.

7. Pasipanodya JG, Mcllleron H, Burger A, Wash PA, Smith P, Gumbo T. Serum drug concentrations predictive of pulmonary tuberculosis outcomes. J Infect Dis. 2013;208(9):1464-73.

8. Cegielski JP, Kurbatova E, van der Walt M, Brand J, Ershova J, Tupasi T, et al. Multidrug-resistant tuberculosis treatment outcomes in relation to treatment and initial versus acquired second-line drug resistance. Clin Infect Dis. 2016;62(4):418-30.

9. Satta G, Lipman M, Smith GP, Arnold C, Kon OM, McHugh TD. Mycobacterium tuberculosis and whole-genome sequencing: how close are we to unleashing its full potential? Clin Microbiol Infect. 2018;24(6):604-9.

10. Sreevatsan S, Pan X, Stockbauer KE, Connell ND, Kreiswirth BN, Whittam TS, et al. Restricted structural gene polymorphism in the mycobacterium tuberculosis complex indicates evolutionarily recent global dissemination. Proc Natl Acad Sci U S A. 1997;94(18):9869-74

11. Sun G, Luo T, Yang C, Dong X, Li J, Zhu Y, et al. Dynamic population changes in mycobacterium tuberculosis during acquisition and fixation of drug resistance in patients. J Infect Dis. 2012;206(11):1724-33.

12. Merker M, Kohl TA, Roetzer A, Truebe L, Richter E, Rüsch-Gerdes S, et al. Whole genome sequencing reveals complex evolution patterns of multidrug-resistant mycobacterium tuberculosis Beijing strains in patients. PLoS One. 2013;8(12):e82551.

13. Operario DJ, Koeppel AF, Turner SD, Bao Y, Pholwat S, Banu S, et al. Prevalence and extent of heteroresistance by next generation sequencing of multidrug-resistant tuberculosis. PLoS One. 2017:12(5):e0176522.

14. Black PA, de Vos M, Louw GE, van der Merwe RG, Dippenaar A, Streicher EM, et al. Whole genome sequencing reveals genomic heterogeneity and antibiotic purification in mycobacterium tuberculosis isolates. BMC Genomics. 2015;16(1):857

15. Eldholm V, Norheim G, von der Lippe B, Kinander W, Dahle UR, Caugant DA et al. Evolution of extensively drug-resistant mycobacterium tuberculosis from a susceptible ancestor in a single patient. Genome Biol. 2014;15(11):490.

16. Bloemberg GV, Keller PM, Stucki D, Stuckia D, Trauner A, Borrell S, et al. Acquired resistance to Bedaquiline and Delamanid in therapy for tuberculosis. N Engl J Med. 2015;373(20):1986-8

17. Lieberman TD, Wilson D, Misra R, Xiong LL, Moodley P, Cohen T, et al. Genomic diversity in autopsy samples reveals within-host dissemination of HIV-associated mycobacterium tuberculosis. Nat Med. 2016;22(12):1470-4.

18. Ford C, Yusim K, loerger T, Feng S, Chase M, Greene M, et al. Mycobacterium tuberculosis--heterogeneity revealed through whole genome sequencing. Tuberculosis (Edinb). 2012:92(3):194-201.

19. Metcalfe JZ, Streicher E, Theron G, Colman RE, Allender C, Lemmer D, et al. Cryptic micro-heteroresistance explains M. tuberculosis phenotypic resistance. Am J Respir Crit Care Med. 2017;196(9):1191-201.

20. Bradley P, Gordon NC, Walker TM, Dunn L, Heys S, Huang B, et al. Rapid antibiotic-resistance predictions from genome sequence data for 
Staphylococcus aureus and mycobacterium tuberculosis. Nat Commun 2015;6:10063.

21. Feuerriegel $S$, Schleusener $V$, Beckert $P$, Kohl TA, Miotto $P$, Cirillo DM, et al. PhyResSE: a web tool delineating mycobacterium tuberculosis antibiotic resistance and lineage from whole-genome sequencing data. J Clin Microbiol. 2015;53(6):1908-14.

22. Coll F, McNerney R, Preston MD, Guerra-Assunção JA, Warry A, Hill-Cawthorne $\mathrm{G}$, et al. Rapid determination of anti-tuberculosis drug resistance from wholegenome sequences. Genome Med. 2015;7(1):51.

23. Doughty EL, Sergeant MJ, Adetifa I, Antonio M, Pallen MJ. Culture-independent detection and characterisation of mycobacterium tuberculosis and $\mathrm{M}$. africanum in sputum samples using shotgun metagenomics on a benchtop sequencer. PeerJ. 2014;2:e585.

24. Bjorn-Mortensen K, Zallet J, Lillebaek T, Andersen AB, Niemann S, Rasmussen $\mathrm{EM}$, et al. Direct DNA extraction from mycobacterium tuberculosis frozen stocks as a Reculture-independent approach to whole-genome sequencing. J Clin Microbiol. 2015;53(8):2716-9.

25. Depledge DP, Palser AL, Watson SJ, Lai IY, Gray ER, Grant P, et al. Specific capture and whole-genome sequencing of viruses from clinical samples. PLoS One. 2011;6(11):e27805.

26. Hanekom M, Streicher EM, Van de Berg D, Cox H, McDermid C, Bosman M, et al. Population structure of mixed mycobacterium tuberculosis infection is strain genotype and culture medium dependent. PLoS One. 2013;8(7): e70178.

27. Martin A, Herranz M, Ruiz Serrano MJ, Bouza E, Garcia de Viedma D. The clonal composition of mycobacterium tuberculosis in clinical specimens could be modified by culture. Tuberculosis (Edinb). 2010;90(3):201-7.

28. Metcalfe JZ, Streicher E, Theron G, Colman RE, Penaloza R, Allender C, et al. Mycobacterium tuberculosis subculture results in loss of potentially clinically relevant heteroresistance. Antimicrob Agents Chemother. 2017;61(11): e00888-17.

29. Jain P, Weinrick BC, Kalivoda EJ, Yang H, Munsamy V, Vilcheze C, et al. Dualreporter Mycobacteriophages (Phi2DRMs) reveal preexisting mycobacterium tuberculosis persistent cells in human sputum. MBio. 2016;7(5):e01023-16.

30. Mukamolova GV, Turapov O, Malkin J, Woltmann G, Barer MR. Resuscitationpromoting factors reveal an occult population of tubercle bacilli in sputum. Am J Respir Crit Care Med. 2010;181(2):174-80.

31. Votintseva AA, Bradley P, Pankhurst L, Del Ojo Elias C, Loose M, Nilgiriwala K, et al. Same-day diagnostic and surveillance data for tuberculosis via whole genome sequencing of direct respiratory samples. J Clin Microbiol. 2017; 55(5):1285-98.

32. Brown AC, Bryant JM, Einer-Jensen K, Holdstock J, Houniet DT, Chan JZ, et al. Rapid whole-genome sequencing of mycobacterium tuberculosis isolates directly from clinical samples. J Clin Microbiol. 2015;53(7):2230-7.

33. Doyle RM, Burgess C, Williams R, Gorton R, Booth H, Brown J, et al. Direct whole genome sequencing of sputum accurately identifies drug resistant mycobacterium tuberculosis faster than MGIT culture sequencing. J Clin Microbiol. 2018;56(8):e00666-18.

34. Nimmo C, Doyle R, Burgess C, Williams R, Gorton R, McHugh TD, et al. Rapid identification of a mycobacterium tuberculosis full genetic drug resistance profile through whole genome sequencing directly from sputum. Int J Infect Dis. 2017;62:44-6.

35. Consortium CR. The GP, Allix-Beguec C, Arandjelovic I, bi L, Beckert P, et al. prediction of susceptibility to first-line tuberculosis drugs by DNA sequencing. N Engl J Med. 2018:379(15):1403-15.

36. Walker TM, Kohl TA, Omar SV, Hedge J, Del Ojo Elias C, Bradley P, et al. Whole-genome sequencing for prediction of mycobacterium tuberculosis drug susceptibility and resistance: a retrospective cohort study. Lancet Infect Dis. 2015:15(10):1193-202.

37. Malik S, Willby M, Sikes D, Tsodikov OV, Posey JE. New insights into fluoroquinolone resistance in mycobacterium tuberculosis: functional genetic analysis of gyrA and gyrB mutations. PLoS One. 2012;7(6):e39754.

38. Coll F, McNerney R, Guerra-Assuncao JA, Glynn JR, Perdigao J, Viveiros M, et al. A robust SNP barcode for typing mycobacterium tuberculosis complex strains. Nat Commun. 2014;5:4812.

39. Sobkowiak B, Glynn JR, Houben R, Mallard K, Phelan JE, Guerra-Assuncao JA et al. Identifying mixed mycobacterium tuberculosis infections from whole genome sequence data. BMC Genomics. 2018;19(1):613.

40. Konstantinidis KT, Tiedje JM. Prokaryotic taxonomy and phylogeny in the genomic era: advancements and challenges ahead. Curr Opin Microbiol. 2007;10(5):504-9.
41. Yang C, Luo T, Shen X, Wu J, Gan M, Xu P, et al. Transmission of multidrugresistant mycobacterium tuberculosis in Shanghai, China: a retrospective observational study using whole-genome sequencing and epidemiological investigation. Lancet Infect Dis. 2017:17(3):275-84.

42. Eldholm V, Monteserin J, Rieux A, Lopez B, Sobkowiak B, Ritacco V, et al. Four decades of transmission of a multidrug-resistant mycobacterium tuberculosis outbreak strain. Nat Commun. 2015;6:7119.

43. Heym B, Alzari PM, Honore N, Cole ST. Missense mutations in the catalaseperoxidase gene, katG, are associated with isoniazid resistance in mycobacterium tuberculosis. Mol Microbiol. 1995;15(2):235-45.

44. Coll F, Phelan J, Hill-Cawthorne GA, Nair MB, Mallard K, Ali S, et al. Genomewide analysis of multi- and extensively drug-resistant mycobacterium tuberculosis. Nat Genet. 2018;50(2):307-16.

45. Sam IC, Drobniewski F, More P, Kemp M, Brown T. Mycobacterium tuberculosis and rifampin resistance, United Kingdom. Emerg Infect Dis. 2006;12(5):752-9.

46. Larsen MH, Biermann K, Tandberg S, Hsu T, Jacobs WR. Genetic manipulation of mycobacterium tuberculosis. Curr Protoc Microbiol 2007; Chapter 10:Unit $10 \mathrm{~A} .2$.

47. Krueger F. TrimGalore. Available from: https://github.com/FelixKrueger/ TrimGalore . [cited 2019 Mar 19].

48. Bushnell B. BBMap. Available from: https://jgi.doe.gov/data-and-tools/ bbtools/. [cited 2019 Mar 19].

49. Picard Tools: Broad Institute. Available from: http://broadinstitute.github.io/ picard/. [cited 2019 Mar 19].

50. Okonechnikov K, Conesa A, Garcia-Alcalde F. Qualimap 2: advanced multisample quality control for high-throughput sequencing data. Bioinformatics. 2016;32(2):292-4.

51. Garrison E, Marth G. Haplotype-based variant detection from short-read sequencing. arXiv preprint arXiv:12073907. 2012.

52. Garrison E. Vcflib. Available from: https://github.com/vcflib/vcflib. [cited 2019 Mar 19].

53. Koboldt DC, Zhang Q, Larson DE, Shen D, McLellan MD, Lin L, et al. VarScan 2: somatic mutation and copy number alteration discovery in cancer by exome sequencing. Genome Res. 2012;22(3):568-76.

54. Page AJ, Taylor B, Delaney AJ, Soares J, Seemann T, Keane JA, et al. SNPsites: rapid efficient extraction of SNPs from multi-FASTA alignments. Microb Genom. 2016;2(4):e000056.

55. Seemann T. snp-dists. Available from: https://github.com/tseemann/snpdists. [cited 2019 Mar 19].

56. Stamatakis A. RAxML version 8: a tool for phylogenetic analysis and postanalysis of large phylogenies. Bioinformatics. 2014;30(9):1312-3.

57. Wood DE, Salzberg SL. Kraken: ultrafast metagenomic sequence classification using exact alignments. Genome Biol. 2014;15(3):R46.

58. Lassalle F, Spagnoletti M, Fumagalli M, Shaw L, Dyble M, Walker C, et al. Oral microbiomes from hunter-gatherers and traditional farmers reveal shifts in commensal balance and pathogen load linked to diet. Mol Ecol. 2018;27(1): 182-95.

59. Camacho C, Coulouris G, Avagyan V, Ma N, Papadopoulos J, Bealer K, et al. BLAST+: architecture and applications. BMC Bioinformatics. 2009:10:421.

Ready to submit your research? Choose BMC and benefit from:

- fast, convenient online submission

- thorough peer review by experienced researchers in your field

- rapid publication on acceptance

- support for research data, including large and complex data types

- gold Open Access which fosters wider collaboration and increased citations

- maximum visibility for your research: over $100 \mathrm{M}$ website views per year

At $\mathrm{BMC}$, research is always in progress.

Learn more biomedcentral.com/submissions 